\title{
LEGENDRE EXPANSION METHODS FOR THE NUMERICAL SOLUTION OF NONLINEAR 2D FREDHOLM INTEGRAL EQUATIONS OF THE SECOND KIND
}

\author{
S. NEMATI* AND Y. ORDOKHANI
}

\begin{abstract}
At present, research on providing new methods to solve nonlinear integral equations for minimizing the error in the numerical calculations is in progress. In this paper, necessary conditions for existence and uniqueness of solution for nonlinear 2D Fredholm integral equations are given. Then, two different numerical solutions are presented for this kind of equations using 2D shifted Legendre polynomials. Moreover, some results concerning the error analysis of the best approximation are obtained. Finally, illustrative examples are included to demonstrate the validity and applicability of the new techniques.

AMS Mathematics Subject Classification : 45G10, 65R20.

Key words and phrases : Two-dimensional Fredholm integral equation, Legendre polynomials, best approximation, polynomial interpolation.
\end{abstract}

\section{Introduction}

There are many works on developing and analyzing numerical methods for solving the 1D Fredholm integral equations of the second kind $[2,5,9,12,10]$. But little work has been done to solve the 2D cases. Papers [14] and [15] are concerned with the numerical solution for linear 2D Fredholm integral equations (2D-FIEs). In [7], an approximate solution of the nonlinear 2D-FIEs was presented by the Nystrom scheme. In [8], the asymptotic expansion for the approximate solution of this kind of equations was considered by the iterated discrete Galerkin method. Alipanah and Smaeili [1] considered the numerical solution of the nonlinear 2D-FIE using Gaussian radial basis function. The authors of [4], presented a numerical solution of the nonlinear 2D-FIEs using rationalized Haar functions.

Received July 4, 2012. Revised February 21, 2013. Accepted March 16, 2013. ${ }^{*}$ Corresponding author.

(c) 2013 Korean SIGCAM and KSCAM. 
In this paper, we consider the following nonlinear 2D-FIE of the second kind

$$
u(x, y)=f(x, y)+\int_{c}^{d} \int_{a}^{b} K(x, y, t, s) g(t, s, u(t, s)) d t d s
$$

where $u(x, y)$ is the unknown function in $\Omega=[a, b] \times[c, d]$, functions $f$ and $K$ are given smooth functions and the function $g$ is given continuous function nonlinear in $u$.

The outline of this paper is as follows: In Section 2, existence and uniqueness of solution are discussed. In Section 3, some properties of 2D shifted Legendre functions are included. In section 4, we give two approximate solutions for equation (1) using 2D shifted Legendre functions. In section 5, we estimate the error of the best approximation for two-variate sufficiently smooth functions. Numerical examples are given in Section 6 to illustrate the accuracy of our methods. Finally, concluding remarks are presented in Section 7.

\section{Existence and uniqueness of solution}

Consider equation (1) with the following assumptions:

(1) $f$ is continuous in $\Omega$,

(2) $K$ is continuous in $Q=\Omega \times \Omega$,

(3) $g$ is continuous in the domain

$$
W=\{(t, s) ; a \leq t \leq b, c \leq s \leq d,|u| \leq \infty\}
$$

and satisfies the Lipschitz condition with respect to its third argument:

$$
|g(t, s, \bar{u})-g(t, s, \overline{\bar{u}})| \leq M|\bar{u}-\overline{\bar{u}}| .
$$

Lemma 2.1. Existence and uniqueness of solution to equation (1) follow by assumptions (1)-(3) and the condition

$$
M_{1} M(b-a)(d-c)<1,
$$

where

$$
M_{1}=\max _{(x, y, t, s) \in Q}|K(x, y, t, s)| .
$$

Proof. It can be proved using Banach's fixed point theorem in a similar method as done in [3], Chapter 5 (for 1D linear Fredholm integral equations).

\section{Properties of $2 \mathrm{D}$ shifted Legendre polynomials}

3.1. Definition and function approximation. 2D shifted Legendre polynomials are defined on $\Omega$ as

$$
\psi_{m n}(x, y)=L_{m}\left(\frac{2}{b-a} x-\frac{b+a}{b-a}\right) L_{n}\left(\frac{2}{d-c} y-\frac{d+c}{d-c}\right), \quad m, n=0,1,2, \ldots,
$$

and are orthogonal with respect to the weight function $\omega(x, y)=1$ such that

$$
\int_{c}^{d} \int_{a}^{b} \omega(x, y) \psi_{m n}(x, y) \psi_{i j}(x, y) d x d y= \begin{cases}\frac{b-a}{2 m+1} \times \frac{d-c}{2 n+1}, & i=m, j=n \\ 0, & \text { otherwise. }\end{cases}
$$


In (2), $L_{m}$ and $L_{n}$ are the well-known Legendre polynomials, respectively of order $m$ and $n$ which are defined on the interval $[-1,1]$ and can be determined with the aid of the following recursive formula [3]:

$$
\begin{aligned}
& L_{0}(x)=1, \\
& L_{1}(x)=x, \\
& L_{m+1}(x)=\frac{2 m+1}{m+1} x L_{m}(x)-\frac{m}{m+1} L_{m-1}(x), m=1,2,3, \ldots
\end{aligned}
$$

We note that $\left\{\psi_{m n}(x, y)\right\}_{m, n=0}^{\infty}$, are total orthogonal basis for the space $L^{2}(\Omega)$ [11]. The inner product in this space is defined by

$$
\langle f(x, y), g(x, y)\rangle=\int_{c}^{d} \int_{a}^{b} f(x, y) g(x, y) d x d y
$$

and the norm is as follows:

$$
\|f(x, y)\|_{2}=\langle f(x, y), f(x, y)\rangle^{\frac{1}{2}}=\left(\int_{c}^{d} \int_{a}^{b}|f(x, y)|^{2} d x d y\right)^{\frac{1}{2}} .
$$

A function $u(x, y)$ defined on $\Omega$ may be expanded as

$$
u(x, y)=\sum_{m=0}^{\infty} \sum_{n=0}^{\infty} c_{m n} \psi_{m n}(x, y)
$$

where

$$
c_{m n}=\frac{\left\langle u(x, y), \psi_{m n}(x, y)\right\rangle}{\left\|\psi_{m n}(x, y)\right\|_{2}^{2}} .
$$

Let $\Pi_{M, N}(\Omega)$ be the space of all polynomials of degree less than or equal to $M$ in variable $x$ and degree less than or equal to $N$ in variable $y$. Then the functions $\left\{\psi_{m n}(x, y)\right\}, m=0,1, \ldots, M, n=0,1, \ldots, N$, form an orthogonal basis for $\Pi_{M, N}(\Omega)$.

If the infinite series in (5) is truncated, then it can be written as

$$
u_{M, N}(x, y)=\sum_{m=0}^{M} \sum_{n=0}^{N} c_{m n} \psi_{m n}(x, y)=C^{T} \psi(x, y),
$$

where $C$ and $\psi(x, y)$ are $(M+1)(N+1) \times 1$ vectors given by

$$
\begin{gathered}
C=\left[c_{00}, c_{01}, \ldots, c_{0 N}, c_{10}, \ldots, c_{1 N}, \ldots, c_{M 0}, \ldots, c_{M N}\right]^{T} \\
\psi(x, y)=\left[\psi_{00}(x, y), \psi_{01}(x, y), \ldots, \psi_{0 N}(x, y), \psi_{10}(x, y), \ldots, \psi_{1 N}(x, y), \ldots\right. \\
\left., \psi_{M 0}(x, y), \ldots, \psi_{M N}(x, y)\right]^{T} .
\end{gathered}
$$

The function $u_{M, N}(x, y)$ is the orthogonal projection of $u(x, y)$ onto the polynomials' space $\Pi_{M, N}(\Omega)$ with respect to the inner product (4) and is the best approximation to $u(x, y)$ (see [11]).

Similarly, any function $K(x, y, t, s)$ in $(\Omega \times \Omega)$ may be expanded in terms of $2 \mathrm{D}$ shifted Legendre polynomials as

$$
K(x, y, t, s) \simeq \psi^{T}(x, y) K \psi(t, s),
$$


where $K$ is a block matrix of the form

$$
K=\left[K^{(i, m)}\right]_{i, m=0}^{M},
$$

in which

$$
K^{(i, m)}=\left[k_{i j m n}\right]_{j, l=0}^{N}, \quad i, m=0,1, \ldots, M,
$$

and $2 \mathrm{D}$ shifted Legendre coefficients $k_{i j m n}$, are given by

$$
k_{i j m n}=\frac{\left\langle\left\langle K(x, y, t, s), \psi_{m n}(t, s)\right\rangle, \psi_{i j}(x, y)\right\rangle}{\left\|\psi_{i j}(x, y)\right\|_{2}^{2}\left\|\psi_{m n}(t, s)\right\|_{2}^{2}}, i, m=0,1, \ldots, M, j, n=0,1, \ldots, N
$$

3.2. Operational matrix of dual. The integration of the product of two vectors $\psi(x, t)$ and $\psi^{T}(x, t)$ using equation (3) is given by

$$
\int_{c}^{d} \int_{a}^{b} \psi(x, t) \psi^{T}(x, t) d x d t=Q=D_{1} \otimes D_{2},
$$

where $D_{1}$ and $D_{2}$ are the operational matrices of $1 \mathrm{D}$ shifted Legendre polynomials, respectively defined on $[a, b]$ and $[c, d]$ of the form

$$
\begin{gathered}
D_{1}=(b-a)\left[\begin{array}{ccccc}
1 & 0 & 0 & \cdots & 0 \\
0 & \frac{1}{3} & 0 & \cdots & 0 \\
0 & 0 & \frac{1}{5} & \cdots & 0 \\
\vdots & \vdots & \vdots & \ddots & \vdots \\
0 & 0 & 0 & \cdots & \frac{1}{2 M+1}
\end{array}\right], \\
D_{2}=(d-c)\left[\begin{array}{ccccc}
1 & 0 & 0 & \cdots & 0 \\
0 & \frac{1}{3} & 0 & \cdots & 0 \\
0 & 0 & \frac{1}{5} & \cdots & 0 \\
\vdots & \vdots & \vdots & \ddots & \vdots \\
0 & 0 & 0 & \cdots & \frac{1}{2 N+1}
\end{array}\right] .
\end{gathered}
$$

In $(7), \otimes$ denotes the Kronecker product defined for two arbitrary matrices $A$ and $B$ as (see [13])

$$
A \otimes B=\left(a_{i j} B\right)
$$

\section{Numerical methods}

In this section, we give two different numerical solutions of equation (1) using $2 \mathrm{D}$ shifted Legendre functions and their properties discussed in previous section.

Method I: Consider nonlinear 2D-FIE of the form (1). Suppose that

$$
G(x, y)=g(x, y, u(x, y)) .
$$

Then equation (1) can be rewritten as

$$
u(x, y)=f(x, y)+\int_{c}^{d} \int_{a}^{b} K(x, y, t, s) G(t, s) d t d s .
$$


We approximate functions in equation (9) by the method mentioned in previous section as

$$
\begin{gathered}
u(x, y) \simeq C^{T} \psi(x, y)=\psi^{T}(x, y) C, \\
f(x, y) \simeq F^{T} \psi(x, y)=\psi^{T}(x, y) F, \\
G(x, y) \simeq G^{T} \psi(x, y)=\psi^{T}(x, y) G, \\
K(x, y, t, s) \simeq \psi^{T}(x, y) K \psi(t, s),
\end{gathered}
$$

where $C$ and $G$ are unknown vectors of order $(M+1)(N+1)$.

Substituting approximations (10)-(13) into equation (9), we obtain

$$
\psi^{T}(x, y) C=\psi^{T}(x, y) F+\psi^{T}(x, y) K\left(\int_{c}^{d} \int_{a}^{b} \psi(t, s) \psi^{T}(t, s) d t d s\right) G .
$$

Using equation (7) in (14), we get

$$
C-F-K Q G=0 .
$$

Moreover, substituting approximations (10) and (12) into equation (8) yields:

$$
g\left(x, y, C^{T} \psi(x, y)\right)=G^{T} \psi(x, y) .
$$

Finally, collocating equation (16) in $(M+1)(N+1)$ points $\left(x_{i}, y_{j}\right)(i=0,1, \ldots, M$, $j=0,1, \ldots, N)$, we get

$$
g\left(x_{i}, y_{j}, C^{T} \psi\left(x_{i}, y_{j}\right)\right)-G^{T} \psi\left(x_{i}, y_{j}\right)=0,
$$

where $x_{i}$ and $y_{j}$ are zeros of $L_{M+1}\left(\frac{2}{b-a} x-\frac{b+a}{b-a}\right)$ and $L_{N+1}\left(\frac{2}{d-c} y-\frac{d+c}{d-c}\right)$ respectively.

Equations (15) and (17) form $2(M+1)(N+1)$ algebraic equations which can be solved in terms of the elements of vectors $C$ and $G$ using the well-known Newton's iterative method. Then we have $\bar{u}(x, y)=C^{T} \psi(x, t)$ as an approximate solution of equation (1).

Method II: In this method, we expand the function $K(x, y, t, s)$ in equation (1) as

$$
K(x, y, t, s) \simeq K^{T}(x, y) \psi(t, s)
$$

where

$$
\begin{aligned}
& K(x, y)=\left[k_{00}(x, y), k_{01}(x, y), \ldots, k_{0 N}(x, y), k_{10}(x, y), \ldots, k_{1 N}(x, y), \ldots\right. \\
& \left., k_{M 0}(x, y), \ldots, k_{M N}(x, y)\right]^{T},
\end{aligned}
$$

and

$$
k_{i j}(x, y)=\frac{\left\langle K(x, y, t, s), \psi_{i j}(t, s)\right\rangle}{\left\|\psi_{i j}(t, s)\right\|^{2}}=\frac{(2 i+1)(2 j+1)}{(b-a)(d-c)} \int_{c}^{d} \int_{a}^{b} K(x, y, t, s) \psi_{i j}(t, s) d t d s .
$$

Considering (8) and approximations (12) and (18), equation (1) can be written as

$$
u(x, y) \simeq f(x, y)+K^{T}(x, y) \int_{c}^{d} \int_{a}^{b} \psi(t, s) \psi^{T}(t, s) d t d s G .
$$


Substituting equation (7) for (19), we obtain

$$
u(x, y) \simeq f(x, y)+K^{T}(x, y) Q G .
$$

Using equations (8), (12) and (20), we get

$$
g\left(x, y, f(x, y)+K^{T}(x, y) Q G\right)=G^{T} \psi(x, y),
$$

Collocating equation $(21)$ at $(M+1)(N+1)$ points $\left(x_{i}, y_{j}\right)(i=0,1, \ldots, M$, $j=0,1, \ldots, N)$ (the points are same as method I), as

$$
g\left(x_{i}, y_{j}, f\left(x_{i}, y_{j}\right)+K^{T}\left(x_{i}, y_{j}\right) Q G\right)=G^{T} \psi\left(x_{i}, y_{j}\right)
$$

Equations (22) form a system of $(M+1)(N+1)$ nonlinear algebraic equations which can be solved using the well-known Newton's iterative method in terms of the elements of vector $G$. Substituting $G$ in equation (20), we find $\hat{u}(x, y)$ as an approximate solution of equation (1). Note that this approximated solution does not belong to polynomials' space $\Pi_{M, N}(\Omega)$, which was introduced in Section 3 .

\section{Estimation of the error}

In this section we are concerned with the error of the approximation of a given two-variate function by its expansion in terms of $2 \mathrm{D}$ shifted Legendre functions. We assume that $f(x, y)$ is a sufficiently smooth function on $\Omega$ and $f_{M, N}(x, y)$ in $\Pi_{M, N}(\Omega)$ is its best approximation. We want to find a bound for $\| f(x, y)-$ $f_{M, N}(x, y) \|_{2}$. For this purpose, consider that $P_{M, N}(x, y)$ is any polynomial of degree $\leq M$ in variable $x$ and degree $\leq N$ in variable $y$. By definition of the best approximation we have

$$
\left\|f(x, y)-f_{M, N}(x, y)\right\|_{2} \leq\left\|f(x, y)-P_{M, N}(x, y)\right\|_{2} .
$$

Inequality (23) is also true for the special case that $P_{M, N}(x, y)$ is the interpolating polynomial to $f$ at points $\left(x_{i}, y_{j}\right)$, where

$$
x_{i}=a+i \frac{b-a}{M}, \quad y_{j}=c+j \frac{d-c}{N}, \quad i=0,1, \ldots, M, j=0,1, \ldots, N .
$$

We have [6]

$$
\begin{aligned}
f(x, y)-P_{M, N}(x, y)= & \frac{\partial^{M+1} f(\xi, y)}{\partial x^{M+1}(M+1) !} \prod_{i=0}^{M}\left(x-x_{i}\right) \\
& +\frac{\partial^{N+1} f(x, \eta)}{\partial y^{N+1}(N+1) !} \prod_{j=0}^{N}\left(y-y_{j}\right) \\
& -\frac{\partial^{M+N+2} f\left(\xi^{\prime}, \eta^{\prime}\right)}{\partial x^{M+1} \partial y^{N+1}(M+1) !(N+1) !} \prod_{i=0}^{M}\left(x-x_{i}\right) \prod_{j=0}^{N}\left(y-y_{j}\right),
\end{aligned}
$$


such that $\xi, \xi^{\prime} \in[a, b]$ and $\eta, \eta^{\prime} \in[c, d]$. Therefore

$$
\begin{aligned}
\left|f(x, y)-P_{M, N}(x, y)\right| \leq & \max _{(x, y) \in \Omega}\left|\frac{\partial^{M+1} f(x, y)}{\partial x^{M+1}}\right| \frac{\prod_{i=0}^{M}\left|x-x_{i}\right|}{(M+1) !} \\
& +\max _{(x, y) \in \Omega}\left|\frac{\partial^{N+1} f(x, y)}{\partial y^{N+1}}\right| \frac{\prod_{j=0}^{N}\left|y-y_{j}\right|}{(N+1) !} \\
& +\max _{(x, y) \in \Omega}\left|\frac{\partial^{M+N+2} f(x, y)}{\partial x^{M+1} \partial y^{N+1}}\right| \frac{\prod_{i=0}^{M}\left|x-x_{i}\right| \prod_{j=0}^{N}\left|y-y_{j}\right|}{(M+1) !(N+1) !} .
\end{aligned}
$$

We would like to derive bounds for the following terms

$$
\prod_{i=0}^{M}\left|x-x_{i}\right|, \quad \prod_{j=0}^{N}\left|y-y_{j}\right| .
$$

We make the change of variables

$$
x=a+\theta \frac{b-a}{M}, \quad y=c+\lambda \frac{d-c}{N}, \quad \theta \in(0, M), \lambda \in(0, N),
$$

thus we obtain,

$$
\begin{aligned}
& \prod_{i=0}^{M}\left|x-x_{i}\right|=\left(\frac{b-a}{M}\right)^{M+1} \prod_{i=0}^{M}|\theta-i|, \\
& \prod_{j=0}^{N}\left|y-y_{j}\right|=\left(\frac{d-c}{N}\right)^{N+1} \prod_{j=0}^{N}|\lambda-j| .
\end{aligned}
$$

Suppose that $k$ and $l$ are integers such that

$$
k<\theta<k+1, \quad l<\lambda<l+1,
$$

therefore, we can write

$$
\begin{aligned}
& \prod_{i=0}^{M}|\theta-i|=|(\theta-k)(\theta-k-1)| \prod_{i=0}^{k-1}|\theta-i| \prod_{i=k+2}^{M}|\theta-i|, \\
& \prod_{j=0}^{N}|\lambda-j|=|(\lambda-l)(\lambda-l-1)| \prod_{j=0}^{l-1}|\lambda-j| \prod_{j=l+2}^{N}|\lambda-j| .
\end{aligned}
$$

The terms $|(\theta-k)(\theta-k-1)|$ and $|(\lambda-l)(\lambda-l-1)|$ give their maximum value respectively at the points $\theta+\frac{1}{2}$ and $\lambda+\frac{1}{2}$. So we can say

$$
\begin{aligned}
& |(\theta-k)(\theta-k-1)| \leq \frac{1}{4}, \\
& |(\lambda-l)(\lambda-l-1)| \leq \frac{1}{4} .
\end{aligned}
$$

Also, using (27), we get

$$
\prod_{i=0}^{k-1}|\theta-i| \leq \prod_{i=0}^{k-1}(k+1-i) \leq(k+1) !
$$




$$
\begin{aligned}
& \prod_{i=k+2}^{M}|\theta-i| \leq \prod_{i=k+2}^{M}(i-k) \leq(M-k) ! \\
& \prod_{j=0}^{l-1}|\lambda-j| \leq \prod_{j=0}^{l-1}(l+1-j) \leq(l+1) ! \\
& \prod_{j=l+2}^{N}|\lambda-j| \leq \prod_{j=l+2}^{N}(j-l) \leq(N-l) !
\end{aligned}
$$

Substituting the estimates in (30)-(35) for (28) and (29) yields:

$$
\begin{aligned}
& \prod_{i=0}^{M}|\theta-i| \leq \frac{1}{4}(M+1) !, \\
& \prod_{j=0}^{N}|\lambda-j| \leq \frac{1}{4}(N+1) ! .
\end{aligned}
$$

Using equations (25), (26), (36) and (37), we obtain

$$
\begin{aligned}
& \prod_{i=0}^{M}\left|x-x_{i}\right| \leq\left(\frac{b-a}{M}\right)^{M+1} \frac{1}{4}(M+1) ! \\
& \prod_{j=0}^{N}\left|y-y_{j}\right| \leq\left(\frac{d-c}{N}\right)^{N+1} \frac{1}{4}(N+1) ! .
\end{aligned}
$$

Substituting bounds in (38) and (39) for (24), we see that the error in two-variate polynomial interpolation satisfies in

$$
\begin{aligned}
\left|f(x, y)-P_{M, N}(x, y)\right| & \leq \frac{1}{4}\left(\frac{b-a}{M}\right)^{M+1} \max _{(x, y) \in \Omega}\left|\frac{\partial^{M+1} f(x, y)}{\partial x^{M+1}}\right| \\
& +\frac{1}{4}\left(\frac{d-c}{N}\right)^{N+1} \max _{(x, y) \in \Omega}\left|\frac{\partial^{N+1} f(x, y)}{\partial y^{N+1}}\right| \\
& +\frac{1}{16}\left(\frac{b-a}{M}\right)^{M+1}\left(\frac{d-c}{N}\right)^{N+1} \max _{(x, y) \in \Omega}\left|\frac{\partial^{M+N+2} f(x, y)}{\partial x^{M+1} \partial y^{N+1}}\right| \\
& =C_{1}\left(\frac{b-a}{M}\right)^{M+1}+C_{2}\left(\frac{d-c}{N}\right)^{N+1}+C_{3}\left(\frac{b-a}{M}\right)^{M+1}\left(\frac{d-c}{N}\right)^{N+1},
\end{aligned}
$$

where

$$
\begin{gathered}
C_{1}=\frac{1}{4} \max _{(x, y) \in \Omega}\left|\frac{\partial^{M+1} f(x, y)}{\partial x^{M+1}}\right|, \\
C_{2}=\frac{1}{4} \max _{(x, y) \in \Omega}\left|\frac{\partial^{N+1} f(x, y)}{\partial y^{N+1}}\right|, \\
C_{3}=\frac{1}{16} \max _{(x, y) \in \Omega}\left|\frac{\partial^{M+N+2} f(x, y)}{\partial x^{M+1} \partial y^{N+1}}\right| .
\end{gathered}
$$

Finally, using (23) we get 


$$
\begin{aligned}
& \left\|f(x, y)-f_{M, N}(x, y)\right\|_{2} \leq\left(\int_{c}^{d} \int_{a}^{b}\left|f(x, y)-P_{M, N}(x, y)\right|^{2} d x d y\right)^{\frac{1}{2}} \\
& \leq\left(\int_{c}^{d} \int_{a}^{b}\left[C_{1}\left(\frac{b-a}{M}\right)^{M+1}+C_{2}\left(\frac{d-c}{N}\right)^{N+1}+C_{3}\left(\frac{b-a}{M}\right)^{M+1}\left(\frac{d-c}{N}\right)^{N+1}\right]^{2} d x d y\right)^{\frac{1}{2}} \\
& =\sqrt{(b-a)(d-c)}\left[C_{1}\left(\frac{b-a}{M}\right)^{M+1}+C_{2}\left(\frac{d-c}{N}\right)^{N+1}+C_{3}\left(\frac{b-a}{M}\right)^{M+1}\left(\frac{d-c}{N}\right)^{N+1}\right] .
\end{aligned}
$$

Remark. In the case that $M=N$ and $\Omega=[0,1] \times[0,1]$, we conclude from (41) that

$$
\left\|f(x, y)-f_{M, N}(x, y)\right\|_{2} \leq\left(C_{1}+C_{2}+C_{3}\left(\frac{1}{M}\right)^{M+1}\right)\left(\frac{1}{M}\right)^{M+1} .
$$

\section{Illustrative examples}

In this section, two examples are given to demonstrate the applicability and accuracy of our methods. In the examples we consider $\Omega=[0,1] \times[0,1]$ and $M=N$. In order to demonstrate the error of the proposed methods, let us introduce the following notations:

$$
\begin{aligned}
& e_{M}(x, y)=\left|u(x, y)-u_{M, M}(x, y)\right| \\
& \bar{e}_{M}(x, y)=\left|u(x, y)-\bar{u}_{M, M}(x, y)\right|, \\
& \hat{e}_{M}(x, y)=\left|u(x, y)-\hat{u}_{M, M}(x, y)\right|,
\end{aligned}
$$

where $u(x, y), u_{M, M}(x, y), \bar{u}_{M, M}(x, y)$ and $\hat{u}_{M, M}(x, y)$ are the exact solution, its best approximation using 2D shifted Legendre functions, the computed solution by Method I and the computed solution by Method II, respectively.

The computations were performed in a personal computer using a processor with $2.20 \mathrm{GHz}$ and the codes were written in Mathematica 8.

Example 6.1. Consider the following nonlinear 2D-FIE [7, 1, 4]

$$
u(x, y)=f(x, y)+\int_{0}^{1} \int_{0}^{1} \frac{x}{1+y}(1+t+s) u^{2}(t, s) d t d s,
$$

where

$$
\frac{1}{(1+x+y)^{2}}-\frac{x}{6(1+y)} \text {. }
$$

Its exact solution is $u(x, y)=1 /(1+x+y)^{2}$. Numerical results are shown in Table 1, Figure 1 and Figure 2.

Example 6.2. Consider nonlinear 2D-FIE as [8]

$$
u(x, y)=f(x, y)+\int_{0}^{1} \int_{0}^{1} \frac{x\left(1-t^{2}\right)}{(1+y)\left(1+s^{2}\right)}(1-\exp (-u(t, s))) d t d s,
$$


TABLE 1. Numerical results for Example 6.1.

\begin{tabular}{|l|lll|lll|}
\hline$M$ & $\left\|e_{M}(x, y)\right\|_{2}$ & $\left\|\bar{e}_{M}(x, y)\right\|_{2}$ & $\left\|\hat{e}_{M}(x, y)\right\|_{2}$ & $\left\|e_{M}(x, y)\right\|_{\infty}$ & $\left\|\bar{e}_{M}(x, y)\right\|_{\infty}$ & $\left\|\hat{e}_{M}(x, y)\right\|_{\infty}$ \\
\hline 1 & $3.29 \times 10^{-2}$ & $3.29 \times 10^{-2}$ & $1.68 \times 10^{-3}$ & $8.62 \times 10^{-2}$ & $8.62 \times 10^{-2}$ & $4.13 \times 10^{-3}$ \\
2 & $6.83 \times 10^{-3}$ & $6.83 \times 10^{-3}$ & $7.62 \times 10^{-5}$ & $2.05 \times 10^{-2}$ & $2.05 \times 10^{-2}$ & $1.86 \times 10^{-4}$ \\
3 & $1.34 \times 10^{-3}$ & $1.34 \times 10^{-3}$ & $2.98 \times 10^{-6}$ & $6.46 \times 10^{-3}$ & $6.46 \times 10^{-3}$ & $7.30 \times 10^{-6}$ \\
4 & $2.55 \times 10^{-4}$ & $2.55 \times 10^{-4}$ & $1.08 \times 10^{-7}$ & $1.39 \times 10^{-3}$ & $1.39 \times 10^{-3}$ & $2.66 \times 10^{-7}$ \\
5 & $4.77 \times 10^{-5}$ & $4.77 \times 10^{-5}$ & $3.82 \times 10^{-9}$ & $3.26 \times 10^{-4}$ & $3.26 \times 10^{-4}$ & $9.36 \times 10^{-9}$ \\
6 & $8.78 \times 10^{-6}$ & $8.78 \times 10^{-6}$ & $1.30 \times 10^{-10}$ & $6.74 \times 10^{-5}$ & $6.74 \times 10^{-5}$ & $3.20 \times 10^{-1}$ \\
7 & $1.53 \times 10^{-6}$ & $1.53 \times 10^{-6}$ & $4.37 \times 10^{-12}$ & $1.41 \times 10^{-5}$ & $1.41 \times 10^{-5}$ & $1.07 \times 10^{-11}$ \\
\hline
\end{tabular}
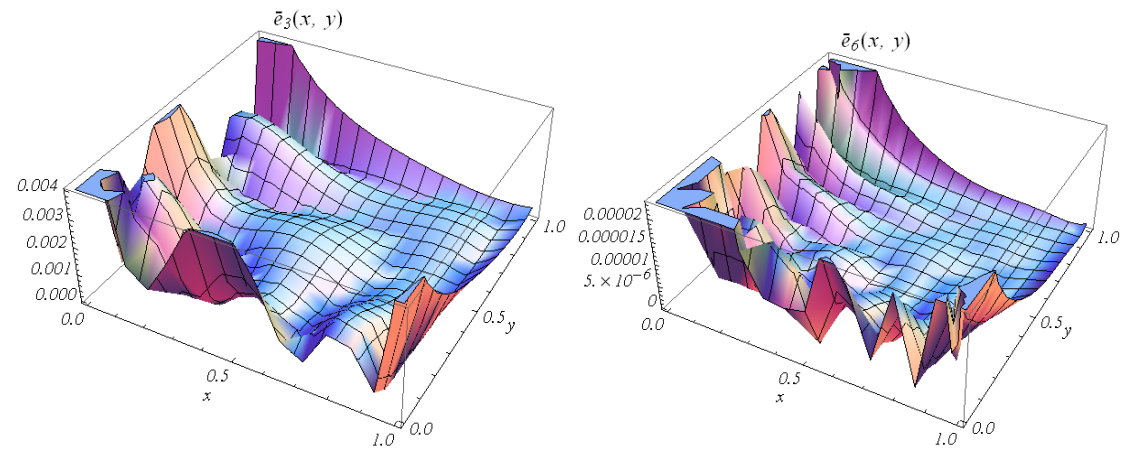

Figure 1. Plot of the absolute error for Example 6.1 using Method I; left: $M=3$, right: $M=6$.
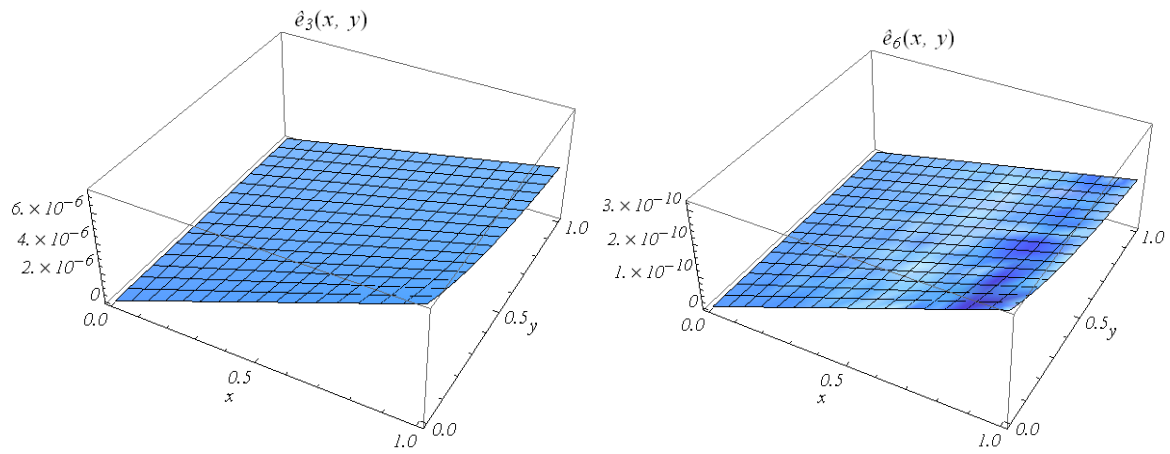

Figure 2. Plot of the absolute error for Example 6.1 using Method II; left: $M=3$, right: $M=6$.

where

$$
f(x, y)=-\operatorname{Ln}\left(1+\frac{x y}{1+y^{2}}\right)+\frac{x}{16(1+y)} .
$$

Its exact solution is $u(x, y)=-\operatorname{Ln}\left(1+\frac{x y}{1+y^{2}}\right)$. Table 2, Figure 3 and Figure 4 give the numerical results for this example. 
TABLE 2. Numerical results for Example 6.2.

\begin{tabular}{|l|lll|lll|}
\hline$M$ & $\left\|e_{M}(x, y)\right\|_{2}$ & $\left\|\bar{e}_{M}(x, y)\right\|_{2}$ & $\left\|\hat{e}_{M}(x, y)\right\|_{2}$ & $\left\|e_{M}(x, y)\right\|_{\infty}$ & $\left\|\bar{e}_{M}(x, y)\right\|_{\infty}$ & $\left\|\hat{e}_{M}(x, y)\right\|_{\infty}$ \\
\hline 1 & $2.44 \times 10^{-2}$ & $2.44 \times 10^{-2}$ & $2.81 \times 10^{-4}$ & $9.90 \times 10^{-2}$ & $9.90 \times 10^{-2}$ & $6.88 \times 10^{-4}$ \\
2 & $2.40 \times 10^{-3}$ & $2.40 \times 10^{-3}$ & $4.23 \times 10^{-6}$ & $1.41 \times 10^{-2}$ & $1.41 \times 10^{-2}$ & $1.03 \times 10^{-5}$ \\
3 & $5.68 \times 10^{-4}$ & $5.68 \times 10^{-4}$ & $1.63 \times 10^{-6}$ & $2.83 \times 10^{-3}$ & $2.83 \times 10^{-3}$ & $3.99 \times 10^{-6}$ \\
4 & $2.01 \times 10^{-4}$ & $2.01 \times 10^{-4}$ & $9.11 \times 10^{-8}$ & $1.11 \times 10^{-3}$ & $1.11 \times 10^{-3}$ & $2.23 \times 10^{-7}$ \\
5 & $2.12 \times 10^{-5}$ & $2.12 \times 10^{-5}$ & $2.14 \times 10^{-9}$ & $1.49 \times 10^{-4}$ & $1.49 \times 10^{-4}$ & $5.26 \times 10^{-9}$ \\
6 & $4.66 \times 10^{-6}$ & $4.66 \times 10^{-6}$ & $1.45 \times 10^{-1}$ & $1.93 \times 10^{-5}$ & $1.93 \times 10^{-5}$ & $3.55 \times 10^{-10}$ \\
7 & $1.60 \times 10^{-6}$ & $1.60 \times 10^{-6}$ & $1.47 \times 10^{-11}$ & $9.05 \times 10^{-6}$ & $9.05 \times 10^{-6}$ & $3.60 \times 10^{-11}$ \\
\hline
\end{tabular}
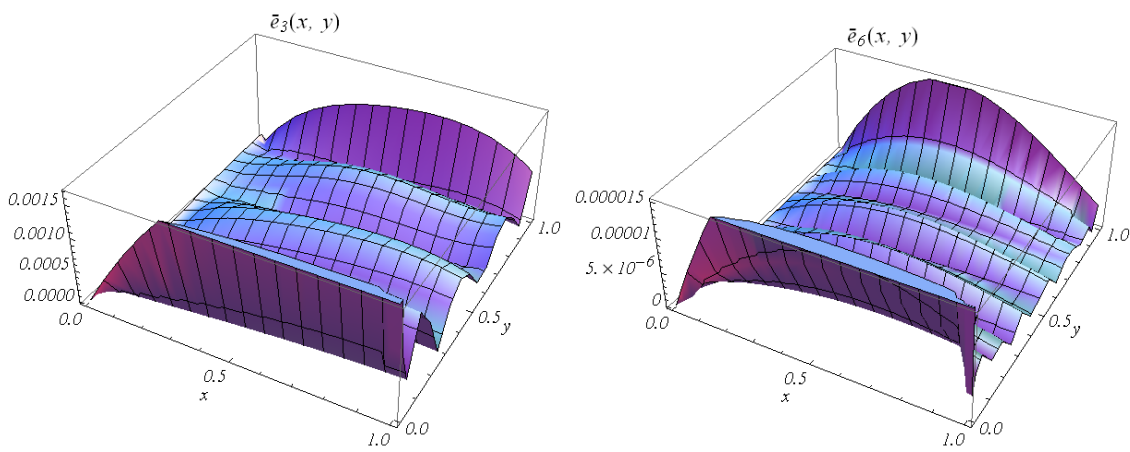

FIGURE 3. Plot of the absolute error for Example 6.2 using Method I; left: $M=3$, right: $M=6$.
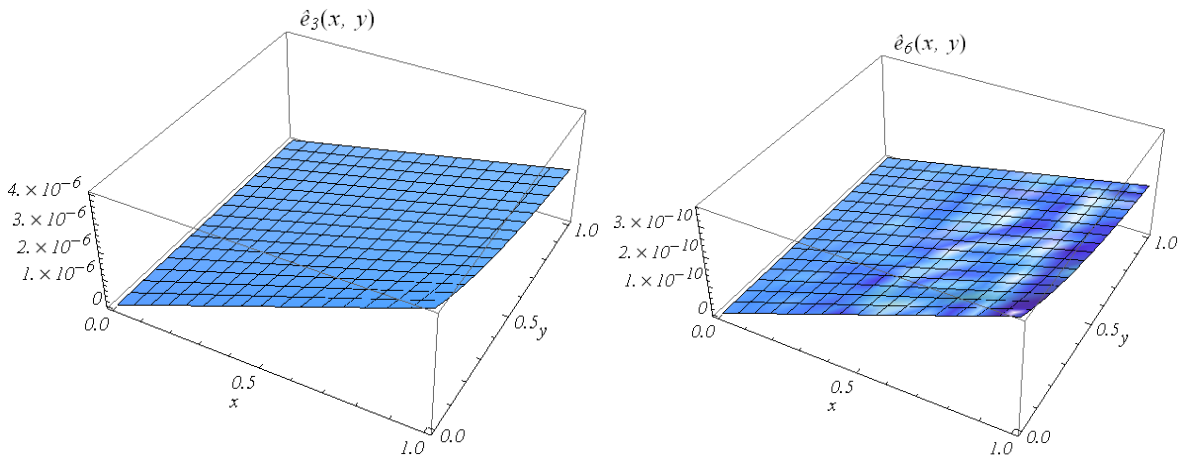

FiguRE 4. Plot of the absolute error for Example 6.2 using Method II; left: $M=3$, right: $M=6$.

\section{Conclusion}

In this paper, we presented two different methods to solve nonlinear 2D-FIEs. The properties of 2D shifted Legendre orthogonal functions together with the collocation method were used to transform the given problem to the solution of nonlinear algebraic equations. Note that the final nonlinear equations can be 
solved using the Newton's iterative method. We gave an error bound when a sufficiently smooth function is approximated in terms of 2D shifted Legendre functions (error of the best approximation of the function). We applied the presented methods on two test problems and compared the results with their exact solutions in order to demonstrate the validity and applicability of the methods. Table 1 and Table 2 show that the numerical solution obtained by Method I has the same error behavior as the error of the best approximation of the exact solution using Legendre functions. Moreover, the required computational effort for Method II is smaller than Method I (the computations can be carried out by a personal computer). It can be found from the tables that Method II has more accurate results than Method I. We leave as future work the error analysis of the proposed methods.

\section{REFERENCES}

1. A. Alipanah, S. Smaeili, Numerical solution of the two-dimensional Fredholm integral equations using Gaussian radial basis function, Journal of Computational and Applied Mathematics, 235(2011), 5342-5347.

2. K. E. Atkinson, The Numerical Solution of Integral Equations of the Second Kind, Cambridge, Cambridge University Press, 1997.

3. K. Atkinson, W. Han, Theoretical Numerical Analysis: a Functional Analysis Framework, Springer-Verlag New York, INC, 2001.

4. E. Babolian, S. Bazm, P. Lima, Numerical solution of nonlinear two-dimensional integral equations using rationalized Haar functions, Communications in Nonlinear Science and Numerical Simulation, 16(2011), 1164-1175.

5. L. M. Delves, J. L. Mohamed, Computational Methods for Integral Equations, Cambridge, Cambridge University Press, 1985.

6. M. Gasea, T. Sauer, On the history of multivariate polynomial interpolation, Journal of Computational and Applied Mathematics, 122(2000), 23-35.

7. H. Guoqiang, W. Jiong, Extrapolation of Nystrom solution for two dimensional nonlinear Fredholm integral equations, Journal of Computational and Applied Mathematics, 134(2001), 259-268.

8. G. Han, R. Wang, Richardson extrapolation of iterated discrete Galerkin solution for two dimensional Fredholm integral equations, Journal of Computational and Applied Mathematics, 139 (2002) 49-63.

9. A. J. Jerri, Introduction to Integral Equations with Applications, INC, John Wiley and Sons, 1999.

10. R. Kress, Linear Integral Equations, Springer-Verlag, 1999.

11. E. Kreyszig, Introductory Functional Analysis with Applications, John Wiley and Sons, 1989.

12. P. K. Kythe, P. Puri, Computational Methods for Linear Integral Equations, Birkhuser, Boston, 2002.

13. P. Lancaster, The Theory of Matrices: with Applications, second ed., Academic Press, New York, 1984.

14. A. Tari, S. Shahmorad, A computational method for solving two-dimensional Linear Fredholm integral equations of the second kind, ANZIAM J., 49(2008), 543-549.

15. W. J. Xie, F. R. Lin, A fast numerical solution method for two dimensional Fredholm integral equations of the second kind, Applied Numerical Mathematics, 59(2009), 17091719. 
S. Nemati PhD, Applied Mathematics.

Department of Mathematics and Computer Science, University of Mazandaran, Babolsar, Iran.

e-mail: so.nemati@yahoo.com

Y. Ordokhani PhD, Applied Mathematics.

Department of Mathematics, Alzahra University, Tehran, Iran.

e-mail: y.ordokhani@alzahra.ac.ir 